\title{
Active deep learning method for the discovery of objects of interest in large spectroscopic surveys ${ }^{\star, \star \star}$
}

\author{
P. Škoda ${ }^{1,2}$, O. Podsztavek ${ }^{2}$, and P. Tvrdík ${ }^{2}$ \\ 1 Astronomical Institute of the Czech Academy of Sciences, Fričova 298, 25165 Ondřejov, Czech Republic \\ e-mail: skoda@sunstel.asu.cas.cz \\ 2 Faculty of Information Technology, Czech Technical University in Prague, Thákurova 9, 16000 Prague 6, Czech Republic \\ e-mail: podszond@fit.cvut.cz, tvrdik@fit.cvut.cz
}

Received 13 June 2019 / Accepted 23 June 2020

\begin{abstract}
Context. Current archives of the LAMOST telescope contain millions of pipeline-processed spectra that have probably never been seen by human eyes. Most of the rare objects with interesting physical properties, however, can only be identified by visual analysis of their characteristic spectral features. A proper combination of interactive visualisation with modern machine learning techniques opens new ways to discover such objects.

Aims. We apply active learning classification methods supported by deep convolutional neural networks to automatically identify complex emission-line shapes in multi-million spectra archives.

Methods. We used the pool-based uncertainty sampling active learning method driven by a custom-designed deep convolutional neural network with 12 layers. The architecture of the network was inspired by VGGNet, AlexNet, and ZFNet, but it was adapted for operating on one-dimensional feature vectors. The unlabelled pool set is represented by 4.1 million spectra from the LAMOST data release 2 survey. The initial training of the network was performed on a labelled set of about 13000 spectra obtained in the $400 \AA$ wide region around $\mathrm{H} \alpha$ by the $2 \mathrm{~m}$ Perek telescope of the Ondřejov observatory, which mostly contains spectra of Be and related early-type stars. The differences between the Ondřejov intermediate-resolution and the LAMOST low-resolution spectrographs were compensated for by Gaussian blurring and wavelength conversion.

Results. After several iterations, the network was able to successfully identify emission-line stars with an error smaller than $6.5 \%$. Using the technology of the Virtual Observatory to visualise the results, we discovered 1013 spectra of 948 new candidates of emission-line objects in addition to 664 spectra of 549 objects that are listed in SIMBAD and 2644 spectra of 2291 objects identified in an earlier paper of a Chinese group led by Wen Hou. The most interesting objects with unusual spectral properties are discussed in detail.
\end{abstract}

Key words. surveys - virtual observatory tools - methods: statistical - techniques: spectroscopic - stars: emission-line, Be line: profiles

\section{Introduction}

The stellar spectral classification, as explained in Gray \& Corbally (2009), is an important astrophysical task of assigning a particular label (mixture of letters and Arabic and Roman numbers), called the spectral class, to each spectrum based on the visual similarities (e.g. presence, strength, and width of the spectral lines of a given element, or a combination of multiple lines). A common automatic procedure (see e.g. Gray \& Corbally 2009, Chap 13.5) uses statistical matching (mainly using $\chi^{2}$ fitting) of a given spectrum with an extensive set of template spectra that may be either synthetic or come from a library of carefully selected stars (called spectral standards). This method is also used in various modifications for the automatic spectral classification of large spectroscopic surveys, such as the Sloan Digital Sky Survey (SDSS; Lee et al. 2008) or Large Sky Area Multi-Object Fiber Spectroscopic Telescope (LAMOST; Wu et al. 2011; Lee et al. 2015).

\footnotetext{
* Catalogues of our emission-line candidates are are only available at the CDS via anonymous ftp to cdsarc.u-strasbg.fr (130.79.128.5) or via http://cdsarc.u-strasbg.fr/viz-bin/ cat $/ \mathrm{J} / \mathrm{A}+\mathrm{A} / 643 / \mathrm{A} 122$

$\star \star$ Based on spectra obtained with $2 \mathrm{~m}$ Perek Telescope of Ondřejov observatory, Czech Republic and archival LAMOST DR2 spectra.
}

A problem arises in many cases when appropriate model of the spectrum is not known and the library used for matching is not rich enough to contain unusual or new types. In addition to this, many types of celestial objects may show complex shapes of only several prominent spectral lines (mainly $\mathrm{H} \alpha$ or other Balmer and Paschen lines) that cover only small parts of the whole spectrum. The integral statistics then fails, and target-tailored methods must be applied to discover such usually rare objects. This is the case of various objects with emission lines that allow us to study a wide range of interesting physical processes.

Pre-main-sequence stars such as young stellar objects and T Tau stars (Reipurth et al. 1996; Kurosawa et al. 2006), or hot stars with expanding envelopes or strong winds show prominent emission lines, as do cataclysmic variables, novae, and even late-type stars with chromospheric activity. See Kogure \& Leung (2007) or Traven et al. (2015) for a comprehensive overview of these cases.

The classical Be stars (Porter \& Rivinius 2003) and the rare class of B[e] stars (Zickgraf 2003) are other cases of well-studied objects with complicated emission-line profiles that often look like symmetric or slightly asymmetric double peaks, sometimes superimposed on absorption lines, depending on their disk geometry (Silaj et al. 2010). The visual classification of their 
profiles (Hanuschik et al. 1988) is a challenging task even on small samples, but it becomes impossible in surveys with millions of spectra.

The classical approach to finding emission lines is to compute integral statistics around their expected positions. It is similar to the standard method of measuring the line equivalent width (Kang \& Lee 2012; Waters \& Hollek 2013).

Such an integral measure based on three-pixel statistics was taken by Lin et al. (2015) on the LAMOST data release 1 (DR1) in order to find strong uprising peaks. This resulted in a catalogue of 203 emission-line stars, 23 of which were identified as classical Be stars and 180 are claimed to be discovered candidates. In order to find double-peak profiles hidden in deep absorption, Hou et al. (2016; hereafter H16) used a more advanced method based on the difference of several statistics with different kernel width. The authors made an extensive analysis of the LAMOST data release 2 (DR2) survey and published a catalogue of 11204 spectra of emission-line stars ${ }^{1}$.

We propose an alternative approach for the discovery of emission-line spectra here based on machine learning of individual shapes of prominent spectral lines. For the sake of simplicity, we limit ourselves to the vicinity of the $\mathrm{H} \alpha$ line. The early attempts on a small sample of good spectra (Škoda \& Vážný 2012; Bromová et al. 2014) have already justified this method, and its application to the LAMOST DR1 (Škoda et al. 2015, 2016) has resulted in the discovery of unknown emission-line candidates. This article describes the first systematic investigation of the LAMOST DR2 using a deep convolutional neural network $(\mathrm{CNN})$ in combination with active learning.

We organised this article as follows. Section 2 describes our active learning method based on CNNs in detail. Section 3 shows the application of the developed method to the discovery of emission-line spectra in the LAMOST DR2. Section 4 discusses the outcomes of the experiment and lists examples of discovered objects of interest. Finally, we conclude in Sect. 5. Furthermore, we compare our method to the non-active learning scenario in Appendix A, and we provide a detailed analysis of the results in Appendix B.

\section{Active deep learning method}

The discovery of objects of interest in large archives of astronomical spectra would be a standard machine learning task if a large and representative labelled data sample of a given archive were available. With such a training set, it would be straightforward to train a supervised learning model and classify the whole archive with high accuracy. However, our experiment in Sect. 3 has shown that if there is no proper training dataset, standard machine learning methods provide poor results with a high rate of both false and missed candidates.

This means that if the training labelled data are not a sufficiently large representation of a spectral archive, for example, when the training set is biased or comes from another, but similar archive, other machine learning approaches need to be developed to obtain reasonable discovery results. We propose and evaluate here an extension of a deep CNN classification method with class balancing and active learning.

The following subsections explain in detail why and how we combined a CNN with a class balancing algorithm and an active learning method. This unified active deep learning workflow allowed us to discover objects of interest (objects with emission-

\footnotetext{
1 http://paperdata.china-vo.org/vac/dr2/

HouEmission2016.tar.gz
}

line spectra) in the LAMOST DR2 altough only a small number of training data were available from a different spectral archive.

\subsection{Deep learning}

Deep learning is a type of machine learning that solves the problem of representational learning by learning a hierarchy of concepts. In representational learning, we try to learn a representation of the data that would facilitate the subsequent learning task. Deep learning allows computers to learn a good data representation by building complicated representations out of more simple ones (LeCun et al. 2015; Goodfellow et al. 2016).

Today, CNNs (LeCun et al. 1989) are the most advanced deep learning method. CNNs started to be recognized when Krizhevsky et al. (2012) achieved a winning top-5 test error rate of $15.3 \%$ on the ImageNet Large Scale Visual Recognition Challenge 2012 (Russakovsky et al. 2015). We wish to take advantage of CNNs because spectra of stellar objects can be viewed as one-dimensional arrays with a single channel, whereas a typical image is a two-dimensional array with usually three RGB channels.

The CNNs are specialised neural networks that use convolution to process data with a grid-like topology. A convolution leverages three essential properties of these biologically inspired networks: sparse interactions (kernels used for convolution with an image have fewer parameters than a fully connected layer), parameter sharing (rather than learning a separate set of parameters, CNNs learn one set for all locations), and equivariance to translation (if an object shifts in the input, its corresponding output shifts by the same distance). Furthermore, a typical $\mathrm{CNN}$ has pooling layers that follow the convolution and activation layers. The pooling layers make the representation invariant to small translations and rotations of the input. This invariance is a useful property for application to spectra because spectral lines might be blue- or red-shifted due to the high radial velocity (Goodfellow et al. 2016).

Deep CNNs have already been successfully applied in astronomy and astrophysics. For example, Aniyan \& Thorat (2017), Domínguez Sánchez et al. (2018), and Alhassan et al. (2018) used CNNs to automate the morphological classification of radio sources. Alger et al. (2018) localised host galaxies for a given radio component with a $\mathrm{CNN}$ using data from experts and crowdsourced training data. Furthermore, George \& Huerta (2018) applied two CNN time-series data to the detection and parameter estimation of gravitational waves from binary black hole mergers. The two CNNs achieved a similar performance as previous advanced methods but were much faster, thus allowing real-time processing. For all these reasons, we decided to develop an active deep learning method with CNNs for the discovery of objects of interest.

\subsection{Class balancing}

When discovering rare objects of interests in large spectroscopic surveys, we face the class imbalance problem (Prati et al. 2009). Labelled spectra of rare objects of interest (hereafter target spectra) will usually be in the minority, in contrast to the labelled spectra of abundant objects (hereafter non-target spectra). Therefore, the labelled training data will tend to be imbalanced. Moreover, target spectra will be in a significant minority in general massive spectral archives (e.g. LAMOST or SDSS).

Our application of the active deep learning, see Sect. 3 for details, revealed exactly the class imbalance problem. The archive of the Ondřejov $2 \mathrm{~m}$ Perek telescope is focused on the 
observation of emission-line stars. Although there is almost the same percentage of single peaks as absorptions, double peaks are still in the minority (see Sect. 3.3). Moreover, there are (at least by order of magnitude) fewer emission-line spectra than standard ones in the LAMOST survey because emission-line objects are rare in the Universe. In these cases, class balancing has shown to be an essential part of workflows and leads to successful performance (e.g. for the necessity of class balancing in astronomy, see de la Calleja et al. 2011 or Lyon et al. 2016, and in medicine, see Rastgoo et al. 2016).

To overcome the fact that CNNs will tend to discriminate the minority classes, we incorporated in our experiments the synthetic minority over-sampling technique (SMOTE) proposed by Chawla et al. (2002). This technique allows enlarging the number of labelled target spectra to the same size as the more abundant non-target spectra.

\subsection{Active learning}

Our experiments have shown that the combination of a CNN and class balancing is still not sufficient for the discovery of objects of interest because the first prediction of candidates delivered a considerable amount of false candidates and featureless noisy spectra. The reason for this failure was an imperfect training dataset. Therefore, we decided to explore active learning (Settles 2009) to circumvent the requirement of good representativeness of labelled samples to exploit the full potential of deep neural networks to discover objects of interest.

Active learning has already shown to be successful in astronomy, for example, in estimating parameters of stellar population synthesis models by Solorio et al. (2005) or for the classification of light curves of variable stars by Richards et al. (2012). Gupta et al. (2016) used active learning to learn a model for photometric data classification from spectroscopic data (the work was extended by Vilalta et al. 2019), and recently, active learning was used to minimise the number of required spectroscopically confirmed labels in preparing training sets for the photometric classification of supernova light curves by Ishida et al. (2019a) and for active anomaly detection in light curves of supernovae by Ishida et al. (2019b). Moreover, active deep learning has been successfully tested in remote sensing by Liu et al. (2017), with further examples reviewed in Yang et al. (2018). To the best of our knowledge, our method represents the first astronomical application of active deep learning.

Active learning is a machine learning technique based on the idea that an algorithm will perform better with fewer training data if it is allowed to choose data for its training. A machine learning algorithm combined with active learning (an active learner) queries unlabelled data examples to be labelled by an oracle (e.g. a human expert).

In the case of large spectra archives, there are huge pools of unlabelled data that can be processed and gathered at once (a so-called pool-based setting in the context of active learning). Spectra are queried selectively from the pool according to an informativeness measure that evaluates all spectra in the pool. Concerning CNNs, the most straightforward approach is to use uncertainty sampling as the query strategy. This strategy selects spectra for which the $\mathrm{CNN}$ provided the least certain labelling because the last layer of the CNN is usually a softmax layer. This layer produces probabilities of classes for each spectrum. Therefore, to query spectra for labelling, we compute the information entropy,

$H=-\sum_{i} p_{i} \ln p_{i}$ where $p_{i}$ is the probability of class $i$, for all the spectra in the pool. Then, the method selects spectra with the highest information entropy.

Because the training of a $\mathrm{CNN}$ can be time-consuming, our method uses so-called batch-mode active learning, which iterates in cycles: an oracle labels a batch of queried samples in each iteration in order to save time and computational resources (training of a CNN). More specifically, the method selects a batch of a previously specified size (e.g. one hundred as in our experiments in Sect. 3.4) from all spectra in the pool, and the oracle visually classifies them. Then, we add all the visually labelled spectra to the training set, so that it contains training data from the previous iterations and newly classified spectra.

Lastly, to decide when to stop the active learning iterative procedure, we need to track the performance of the CNN. The obvious possibility is to estimate a performance measure and stop learning when a plateau is reached (e.g. when adding newly labelled spectra would not increase the performance measure of the $\mathrm{CNN}$ ).

When a large pool of unlabelled samples contains a negligible number of target spectra, it is reasonable to estimate precision, defined as

precision $=\frac{\mathrm{TP}}{\mathrm{TP}+\mathrm{FP}}$

where TP (true positive) is the number of correctly predicted target spectra, and FP (false positive) is the number of incorrectly predicted target spectra. In the case of precision, we can expect that a random sample of spectra classified into target classes will contain the true target spectra. On the other hand, a random sample of all spectra or non-target spectra will probably contain only non-target spectra. Therefore, an estimation of any performance metric based on such random samples will not yield a useful result. For example, an estimate of accuracy, which has to be based on a random sample of all spectra, will almost certainly be 1 or very close to it. Moreover, when discovering rare objects, we are not interested in accuracy, but rather in precision and recall $^{2}$. However, the estimation of recall faces the same problem as the estimation of accuracy.

For this reason, we cannot have any randomly sampled performance estimation set fixed for all iterations. However, we have to sample a new random sample in every iteration as the set of predicted target spectra is changing.

In summary, our active deep learning method takes the labelled data as the initial training set and balances it. Having a balanced training set, we train the $\mathrm{CNN}$ and use the trained CNN to classify all the unlabelled pool of spectra. Then, we use the uncertainty sampling query strategy to obtain a batch of samples for labelling by an oracle that labels all the samples in the batch. The labelled samples are taken out of the unlabelled pool and placed into the labelled training set. Now, we repeat these steps until the performance of our CNN is satisfactory. When we are satisfied with the CNN performance, the unlabelled samples that were lastly predicted as target ones become new candidates of emission-line stars. Finally, we move the samples labelled by the oracle as target from the training set to the candidate set. The flowchart in Fig. 1 illustrates the whole algorithm of our active deep learning method.

\footnotetext{
2 Recall is the ratio of correctly predicted target spectra and all target spectra.
} 


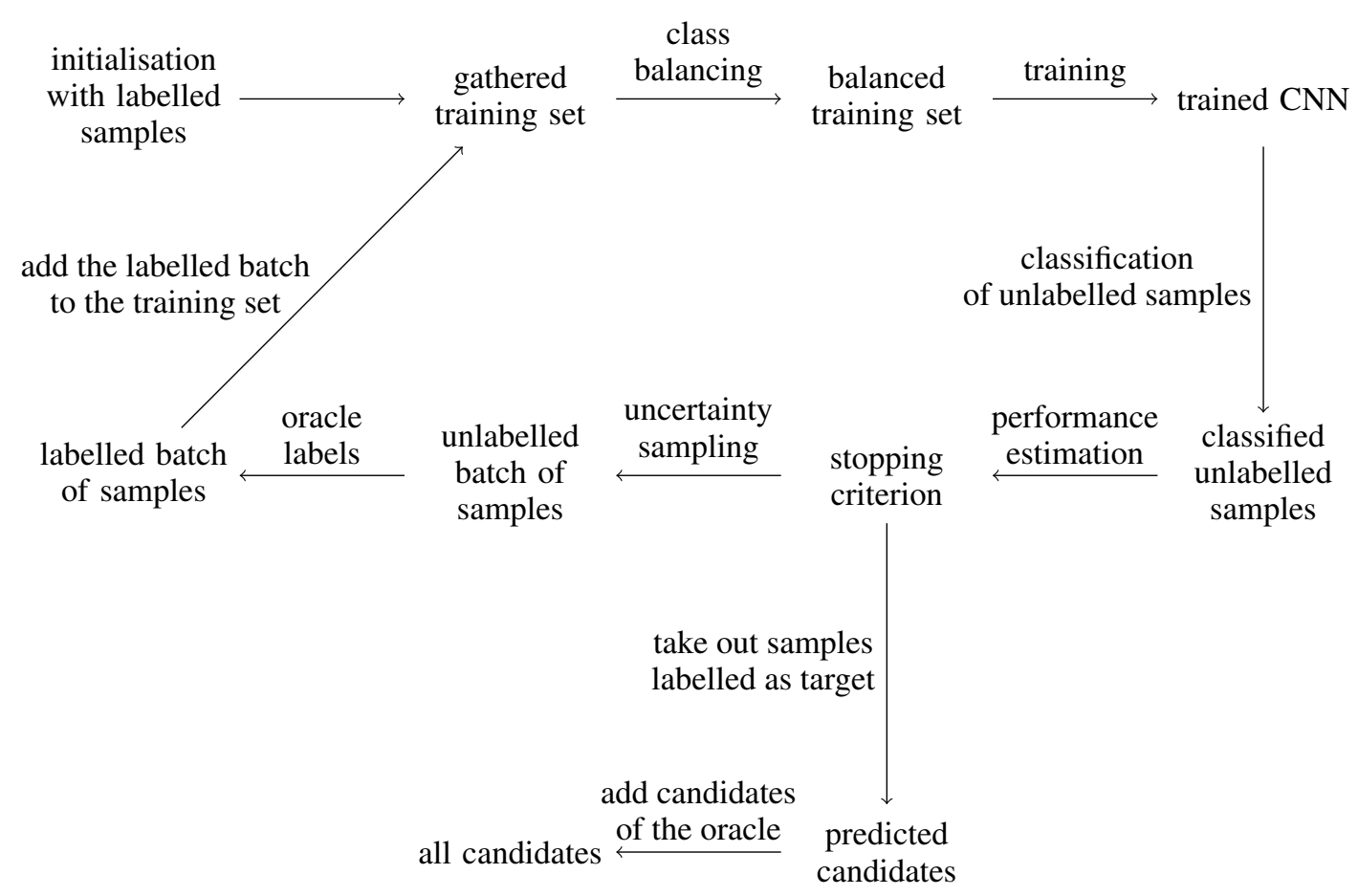

Fig. 1. Flowchart of our active learning method. First, the algorithm is initialised only with labelled training data, the CNN is trained, and unlabelled spectra are classified. Then, uncertainty sampling selects the spectra that the network is least certain for. Finally, these spectra are labelled by an oracle, are added to the training set, and a new training iteration starts. When the performance is satisfactory, samples classified into target classes are taken as candidates and are extended with samples classified into target classes by the oracle.

\section{Experiments}

To illustrate the application of our active deep learning method, we have performed experiments with the discovery of objects with signatures of $\mathrm{H} \alpha$ emission in the LAMOST DR2 survey using labelled data from the Ondřejov $2 \mathrm{~m}$ Perek telescope. The following sections describe the data, the data preparation, the classes of interest, and our method application.

\subsection{Input data}

The archive of spectra obtained with $700 \mathrm{~mm}$ camera in the Coudé spectrograph of the $2 \mathrm{~m}$ Perek telescope at the Ondřejov observatory of the Astronomical Institute of the Czech Academy of Sciences is a unique source of spectra of emission-line stars (mostly Be and $\mathrm{B}[\mathrm{e}]$ stars, stars with strong winds and several novae). This continuously growing archive (hereafter CCD700), currently contains about 17000 spectra, the majority of which (more than 13000) are exposed in spectral range 6250-6700 with a spectral resolving power of about 13000 . The standard IRAF procedure (Škoda \& Šlechta 2002) reduces the spectra, including the calibration in air wavelengths and heliocentric correction.

The LAMOST telescope has delivered one of the currently largest collections of optical spectra. Four thousand fibres positioned by micro-motors feed 16 LAMOST spectrographs. Its publicly available DR2 contains over four million spectra with a spectral resolution power of about 1800 , covering the range 3690-9100 ̊ (Luo et al. 2016). The LAMOST pipeline (Wu et al. 2011) automatically assigns an estimated spectral class to the spectra. However, the pipeline uses classification mostly based on the global shape and integral properties of a spectrum in given band-passes using a set of predefined templates. The local features (e.g. detailed line profiles) are ignored. Strong narrow emissions can even be rejected by the pipeline as possibly spoiled pixels. Therefore, we did not use the assigned spectral classes. Hereafter we call the set of all unlabelled LAMOST DR2 spectra the LAMOST pool. The spectral axis of the FITS files in the LAMOST archive are expressed in the logarithm of the vacuum wavelength.

\subsection{Data preprocessing}

A common assumption in machine learning is that training data (in our work, the CCD700 data) and the data of interest (the LAMOST pool) are from the same probability distribution (Pan \& Yang 2010). However, in this work, we are interested in the classification of the LAMOST pool using the training set from the Ondřejov spectrograph, which contains mostly emission spectra. This means that the training set is highly biased. The distribution mismatch between the training data and the data of interest is a well-known problem in machine learning and is called domain adaptation (Glorot et al. 2011).

Using the technology of the Virtual Observatory (see Appendix B.1) for cross-matching, we have identified only 22 spectra that were observed both by the Ondřejov $2 \mathrm{~m}$ Perek Telescope and by LAMOST. Only a few (e.g. BT CMi, HD 53 416, or V395 Aur) of them show emission lines. The lack of labelled training spectra in the LAMOST pool prevents the usage of supervised training. To use the CCD700 spectra as our training set, we therefore applied a domain transfer to the CCD700 spectra (based on optical engineering procedures), so that they will look as if they were exposed with the LAMOST spectrograph. Taigman et al. (2017) claimed that domain transfer is useful when solving the domain adaptation problem. 

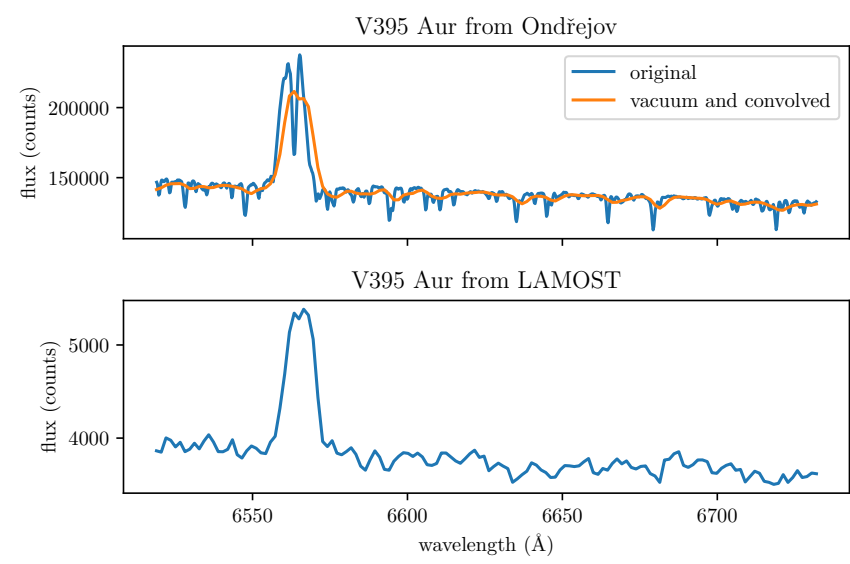

Fig. 2. Comparison of a LAMOST spectrum with a Ondrejov CCD700 spectrum converted into the LAMOST lower resolution and vacuum wavelengths.

Firstly, we applied air-to-vacuum wavelength conversion to the CCD700 spectra using formulas provided in Heiter (2014) because spectra from the CCD700 archive are in air wavelengths, but the LAMOST spectra use vacuum wavelengths. Additionally, we converted the vacuum wavelengths of spectra from the LAMOST pool from the logarithmic into linear scale.

Secondly, because the CCD700 spectra have a higher spectral resolution than the LAMOST spectra, we applied the spectral resolving power degradation to the CCD700 spectra, roughly approximated by the convolution with the Gaussian kernel of a given pixel width to reduce the high-resolution details. Comparison figures of simulated spectra from CCD700 and the LAMOST pool of all 22 objects mentioned above showed that the standard deviation of seven-pixel value works best. Figure 2 shows the comparison of an Ondřejov spectrum, a cross-matched LAMOST spectrum, and the preprocessed spectrum.

Next, the $\mathrm{CNN}$ requires a vector of features as an input. To have the same features for all spectra, they need to be resampled to obtain the measurement in the same wavelengths across all spectra. We decided to use a linear interpolation (using the linear interpolation function of the NumPy library) to 140 uniformly distributed wavelength points in the spectral range between 6519 and $6732 \AA$. We used this number of points because the LAMOST spectra mostly have this number of measurements in the given range. We derived the range from the fact that our classification is based on the $\mathrm{H} \alpha$ line and most of the CCD700 spectra are exposed between these wavelengths. This range also contains He I $6678 \AA$ line, which is important in Be stars. Having resampled all spectra in the same wavelength points, we can create a design matrix required for learning, where rows are 140-dimensional feature vectors of spectra and columns contain fluxes in specified wavelengths.

The last step of preprocessing is the min-max normalisation of the spectral flux into a unit-less range $[-1,1]$ using the equation

$\boldsymbol{x}^{\prime}=2 \frac{\boldsymbol{x}-\min (\boldsymbol{x})}{\max (\boldsymbol{x})-\min (\boldsymbol{x})}-1$,

where $\boldsymbol{x}$ is an input not-scaled spectrum, and $\boldsymbol{x}^{\prime}$ is a scaled spectrum. Thus, each spectrum has a maximum flux of value 1 and a minimum of value -1 . We applied this preprocessing procedure for two reasons: we would like to classify the spectra according to their shapes (this procedure effectively suppresses the differences in intensities), and it obtains the value in the comfortable
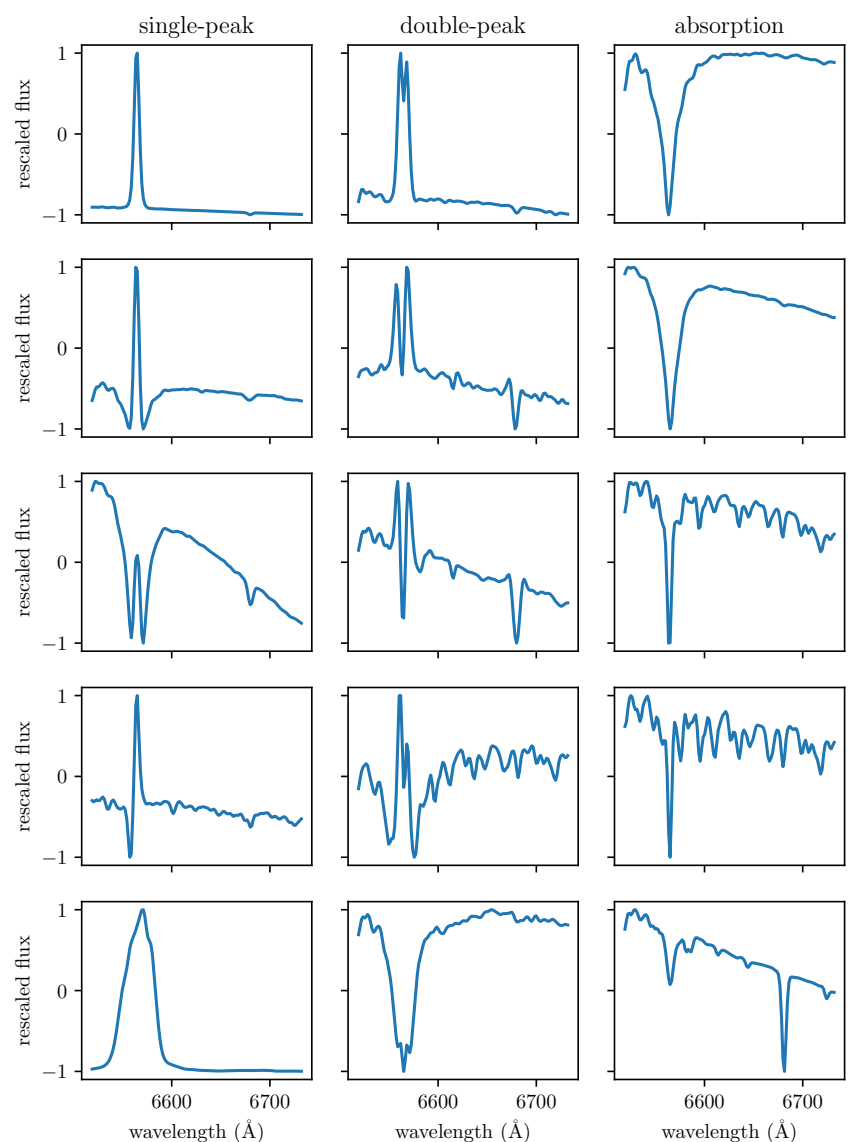

Fig. 3. Examples of spectra from all three classes in the Ondřejov dataset.

small-valued range that is suitable for a neural network training (this is not a feature scaling, but a scaling across each spectrum).

\subsection{Classification}

In the next step, the preprocessed CCD700 spectra were classified by Podsztavek (2017) according the visual shape of the $\mathrm{H} \alpha$ into three classes: single peak, double peak, and absorption. The labelled spectra resulted in a dataset of 12936 labelled spectra (hereafter the Ondřejov dataset ${ }^{3}$ ) that were suitable for machine learning. The counts of spectra in classes are the following:

- Single peak: 5301 spectra (40.98\%),

- Double peak: 1533 spectra $(11.85 \%)$, and

- Absorption: 6102 spectra (47.17\%).

Figure 3 displays representatives of each class. In both single-peak and double-peak spectra the $\mathrm{H} \alpha$ line is in emission, and the difference between the two classes is in the number of peaks, which are clearly visible in the spectrum. Spectra in the single-peak and double-peak target classes are the target emission spectra of our interest, and as expected, their number is smaller than the number of non-target absorption spectra, which are not interesting for us.

The Ondřejov dataset contains only well-exposed spectra, while the LAMOST pool contains many noisy spectra with instrumental and reduction artefacts, spectra without peaks or absorption, and spectra with low signal-to-noise ratio. During our experiment, we placed all these spectra into the non-target

3 https://doi.org/10.5281/zenodo. 2640970 


\begin{tabular}{|c|}
\hline input (140 pixel spectrum) \\
\hline conv3-64 \\
\hline conv3-64 \\
\hline maxpool2 \\
\hline conv3-128 \\
\hline conv3-128 \\
\hline maxpool2 \\
\hline conv3-256 \\
\hline conv3-256 \\
\hline maxpool2 \\
\hline fc-512 \\
\hline dropout \\
\hline fc-512 \\
\hline dropout \\
\hline softmax \\
\hline
\end{tabular}

Fig. 4. Architecture of our CNN. Convolutional layers are marked as conv3, where the number 3 means the size of the filter in pixels. The mark is followed by a dash and a number that specifies the count of filters. maxpool2 are max-pooling layers with pool size 2, stride 2, and no padding. fc -512 denotes a fully connected layer with 512 units, and softmax is the output layer. Dropout layers (Srivastava et al. 2014) with the hyperparameter set to the value 0.5 are used as regularisers.

uninteresting class. Therefore the non-target not interesting class contains bad and absorption spectra, which are both uninteresting for us.

\subsection{Application of active deep learning}

When the data were ready, we applied our method. We chose the architecture of a CNN as developed in previous work that proved to be working well (see Podsztavek 2017). This CNN architecture was inspired primarily by VGGNet (Simonyan et al. 2015), AlexNet (Krizhevsky et al. 2012), and ZFNet (Zeiler et al. 2014). However, these CNNs were designed to process multi-channel two-dimensional images. We therefore adapted the architecture for our one-dimensional data (replace two-dimensional convolutions with one-dimensional convolutions). After several experiments, we converged to the architecture shown in Fig. 4. This CNN was implemented using TensorFlow (Abadi et al. 2015) through the Keras (Chollet 2015) high-level interface and was run on an NVIDIA GTX980 GPU (4 GB memory, 2048 CUDA cores). The network was trained with the Adam optimiser (Kingma et al. 2015) in the default setting of Keras. The best-found weights were restored at the end of each training. We stopped the training when the categorical cross-entropy loss function was not improved by at least $10^{-4}$ during the last ten iterations.

After we trained the $\mathrm{CNN}$ with the Ondřejov dataset (the initial training set) balanced with SMOTE, we used the model to predict classes and probabilities of classes for all spectra in the LAMOST pool. From all the classified spectra, a batch of 100 spectra with the highest information entropy computed from the probabilities of classes was selected (the uncertainty sampling strategy), visually reviewed by us (in the role of the oracle), and

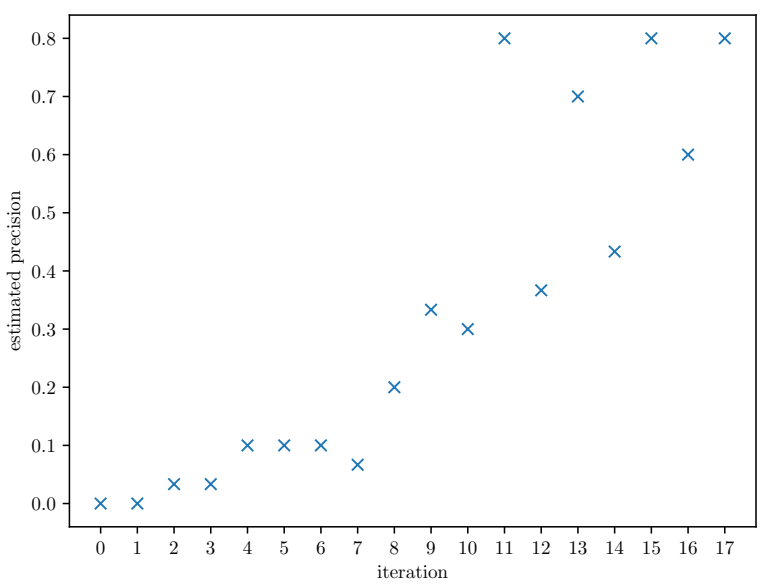

Fig. 5. Estimated precision from a sample of 30 single-peak and doublepeak spectra for each iteration (the zeroth iteration is estimated when the $\mathrm{CNN}$ is trained only with the initial Ondřejov dataset).

classified. Then, all the 100 visually labelled spectra were moved to the training set and removed from the LAMOST pool. Hence, after the first iteration, the training set contained the spectra from the Ondřejov dataset and 100 new spectra from the LAMOST pool.

To track the performance of our $\mathrm{CNN}$, we decided to estimate the precision (the ratio of correctly predicted single-peak and double-peak spectra in all predicted target spectra) in each iteration because of the reasons stated in Sect. 2.3. Therefore we randomly selected 30 spectra classified into single-peak and double-peak (target spectra) classes from the LAMOST pool (hereafter the performance estimation sample). The size of 30 was chosen as a good trade-off between confidence and the demands of visual verification. Then we manually labelled the performance estimation sample and compared our labels with the labels predicted by our CNN. Thus we estimated the precision after each iteration. The performance estimation sample of 30 spectra functions as a test set. In a standard machine learning scenario, a test set is a random sample of all unseen data that could be put into the $\mathrm{CNN}$. In our case, all possible data for our $\mathrm{CNN}$ are in the so far unlabelled LAMOST pool. Therefore, the performance estimation sample will provide an unbiased estimate of precision. We would like to point out that the manual labelling of the performance estimation sample is different from the labelling by the oracle. The labels of the performance estimation sample are forgotten after the precision estimation, and the spectra are left in the LAMOST pool.

Finally, we stopped our experiment in the 17th iteration when the estimated precision reached more than the predefined threshold (in our case $80 \%$ ) for the third time. We chose the values of these parameters as a trade-off between time and performance requirements, and it can be chosen differently for different datasets. Figure 5 displays the precision of our CNN over 17 active learning iterations.

Because the training of our $\mathrm{CNN}$ was time-consuming, we sped up the method by training the CNN during the active learning phase for a smaller number of epochs. Then, after the active learning phase, we ran the Adam optimisation algorithm of the $\mathrm{CNN}$ for a longer time (the training was stopped when the loss function did not improve by $10^{-5}$ during 100 training iterations) to ensure that good convergence was achieved, and thus fewer false candidates will be produced. In the following text, we refer to this step as long training. 
Table 1. Partial confusion matrix of the final classification of our experiment (excluding candidates found by the oracle).

\begin{tabular}{cccc}
\hline \hline \multirow{2}{*}{$\begin{array}{c}\text { Predicted } \\
\text { class }\end{array}$} & \multicolumn{3}{c}{ Actual class } \\
\cline { 2 - 4 } & Single peak & Double peak & Uninteresting \\
\hline Single peak & $97.5 \%(3484)$ & $1.5 \%(53)$ & $1.0 \%(37)$ \\
Double peak & $3.1 \%(18)$ & $93.4 \%(548)$ & $3.6 \%(21)$ \\
\hline
\end{tabular}

Notes. The numbers show the percentage and counts (in brackets) of correctly predicted spectra of all spectra predicted for a given class. The 4161 spectra in this table are all the candidates predicted as single or double peaks after the long training. After we visually reviewed al of them, we found that 58 of candidates are uninteresting spectra (37 predicted as single peaks and 21 predicted as double peaks). The target classes also include some misclassification: 53 double peaks are classified as single peaks, and 18 single peaks are classified as double peaks. We note that we were unable to compute the last row of the uninteresting class because it would mean visually classifying all the four million spectra that are predicted as uninteresting.

\section{Results}

Our method identified 4379 candidate spectra with signatures of emission-line profiles including candidates found by the oracle in all the 4136482 LAMOST DR2 spectra. The last CNN predicted 3574 spectra as single-peak and 587 as double-peak profiles, while the oracle found 157 single-peak candidates and 61 double-peak candidates during the active learning phase. As explained earlier, it also includes absorption profiles with small visible disturbances that may be caused by additional circumstellar emissions. After visual inspection (see Appendix B) of the predicted candidates, we rejected 58 as bad (partly destroyed, noisy, or with pure absorption profiles) and computed the partial confusion matrix in Table 1 . Finally, we had a set of 4321 spectra of about ${ }^{4} 3788$ individual objects.

This set includes 2644 spectra of 2291 objects that have been found previously by H16, and 664 new spectra of 549 objects that are listed in SIMBAD (which were not found by H16). Our method proved to be reliable (with an error smaller than $6.5 \%$ ) because most of the candidates are classified in SIMBAD as various cases of emission-line objects, such as cataclysmic variables, young stellar objects, dwarf novae, symbiotic binaries, IR excess objects from IRAS, classic Be stars and HAEBe stars. In addition, our method found 1013 spectra of 948 new objects that are neither known in SIMBAD nor discovered by H16.

The newly discovered objects span almost all spectral classes as assigned by the LAMOST stellar pipeline, but also many unclassified ones. The visual inspection has confirmed that all of them have signatures of emission in their line profiles. Some have even prominent strong emissions. These include three supernovae candidates, an unknown Wolf-Rayet star, and many Be stars and young stellar objects. Section 4.2 shows examples of our findings.

Moreover, through the visual preview of candidates, several normal and Seyfert galaxies and a high-velocity star, LAMOST HVS1, were also identified. Lastly, we compared our active deep learning method to the dual non-active learning scenario in Appendix A. The comparison shows the significant gain of our active deep learning method.

\footnotetext{
4 The exact number of individual objects is difficult to estimate because of cross-matching problems, as explained in Appendix B.2.
}

\subsection{On-line material}

The final catalogues of spectra of all our emission-line candidates obtained by active deep learning are available at the CDS (see the footnote on the title page), and they are also stored in the science cloud in the Zenodo repository ${ }^{5}$ (Škoda et al. 2019) for further investigation. HTML tables list the spectra that were known by H16, spectra of new objects that we were able to crossmatch with SIMBAD, and all our new so far unknown emissionline spectra. For the sake of completeness, we also show the table of spectra that were visually proved to be in some way broken, extremely noisy, or lacked emission signatures. All of these tables also contain direct links to the CDS Vizier repository of LAMOST DR2, where the particular spectrum may be interactively plotted. Furthermore, there are the CSV versions (suitable for spreadsheets) and VOTable format for further analysis in VO tools, such as Aladin, Topcat, or SPLAT-VO. The electronic PDF version of this paper includes electronic links to various public resources, such as LAMOST or SDSS archives of spectra, DSS images of the sky, or detailed description of objects in SIMBAD including links to our previews stored at Zenodo ${ }^{6}$ as well.

\subsection{Examples of rare interesting objects}

The important research result is also a list of objects with unusual spectra properties. Some objects may have been caught just during a LAMOST observation in a particular evolutionary phase, or we might have witnessed the outburst of an unknown nova or supernova. Many such spectra correspond to known SIMBAD objects, but the SIMBAD class of such objects may be different from what we see. Very interesting objects were also found by checking the bad (noisy or artefact-contaminated) spectra in detail. For example, a part of the spectral range could be destroyed, but the remaining part with a dominant line profile may be still intact.

\subsubsection{New emission-line stars}

Our candidate list also includes 1013 spectra of 948 objects that are neither cross-matched with SIMBAD nor listed by H16. They cover a wide range of line profile shapes belonging to new so far unknown Be stars, T Tau stars, cataclysmic variables, close binaries, symbiotic stars etc. Figures 6 and 7 show randomly selected examples of interesting spectra of each target class.

Some stars have quite peculiar spectra that exhibit quite complicated profiles in multiple lines. This promises interesting physical conditions.

Spectrum spec-56661-GAC061N34B1_sp07-028 of object LAMOST J040901.83+323955.6 displays dominating emission in N III $4644 \AA$ and He II $4686 \AA$ lines, which suggests that this might be a Wolf-Rayet WN star (Walborn \& Fitzpatrick 2000). See Fig. 8.

Spectrum spec-56699-GAC085N52V3_sp15-178 of object LAMOST J053944.81+531825.7 shows quite a bad merging of LAMOST red and blue spectrographs. This was done by the reduction pipeline (therefore it is listed in the table of bad candidates), but the red part shows P Cyg and inverse P Cyg profiles in several lines and emission combined with absorption at $7610 \AA$ (see Fig. 9). In the DSS2 survey, a strange object with the signature of an edge-on ring (resembling Saturn) lies at the given position. However, the object is resolved into three in-line

\footnotetext{
5 https://doi.org/10.5281/zenodo. 3241520

6 https://doi.org/10.5281/zenodo. 3236165
} 

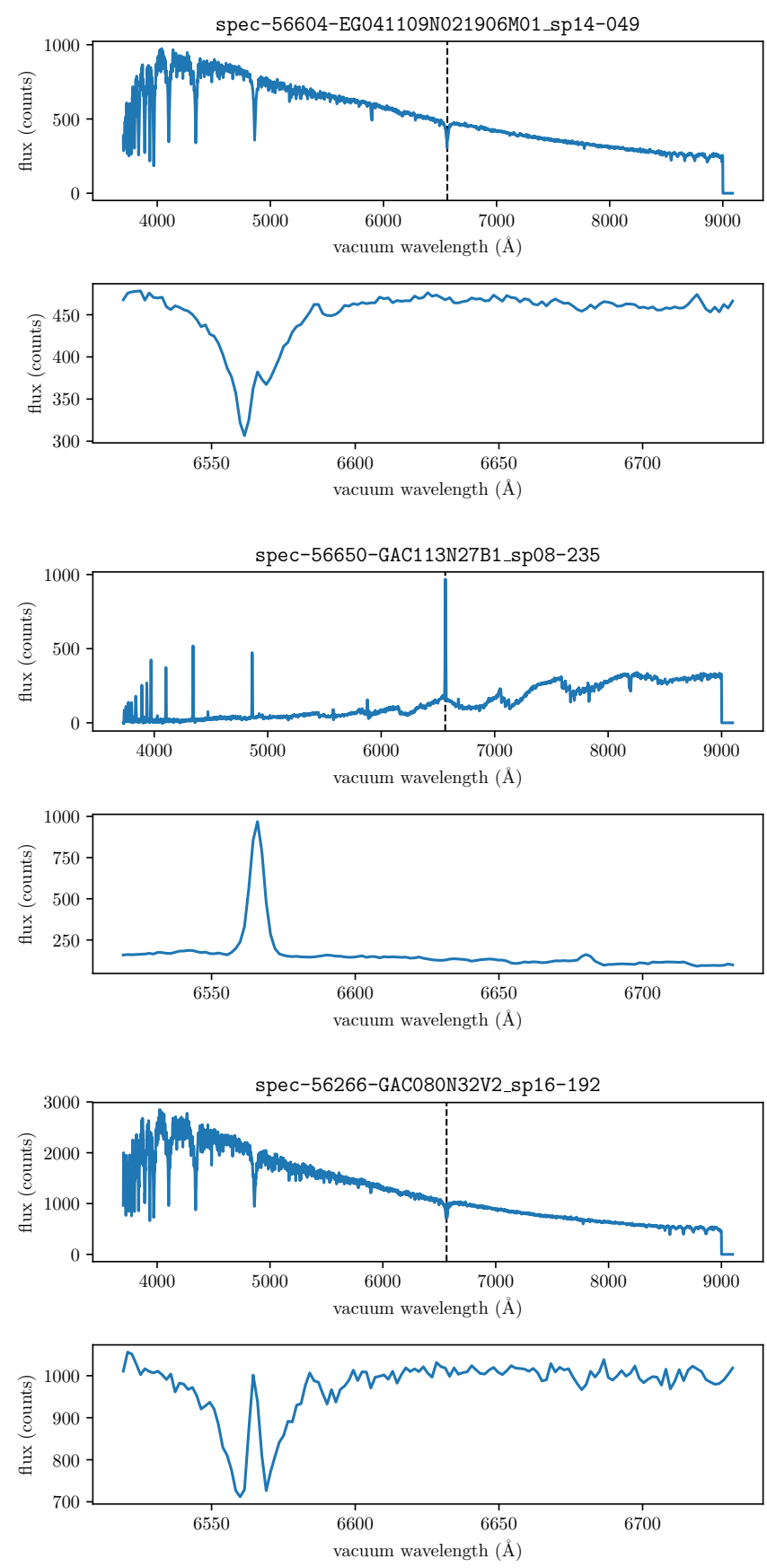

Fig. 6. Examples of spectra classified as single peaks.

stars in the PanSTARRS-1 survey. Both satellite stars are perfectly aligned in a straight line with the bright central object, and they are separated by almost exactly $6.7^{\prime \prime}$ from its centre. The configuration did not change since the PanSTARRS exposures (secured multiple times in years 2011 to 2014) up to now, which we confirmed on 15 April 2020 by the exposure of the newly commissioned photometric camera of the $2 \mathrm{~m}$ Perek telescope at Ondřejov observatory. The symmetrical configuration deserves further investigation as it may be an effect of gravitational lensing.

Spectrum spec-56202-EG042015S023742V01_sp15-180 in Fig. 10 is very similar, including the same bug in joining red and blue spectrograph, showing the object LAMOST J041919. 80-020211.6, which is neither known to SIMBAD nor exposed by the SDSS. It has two emissions at $5790 \AA$ and $5820 \AA$, the $\mathrm{H} \alpha$
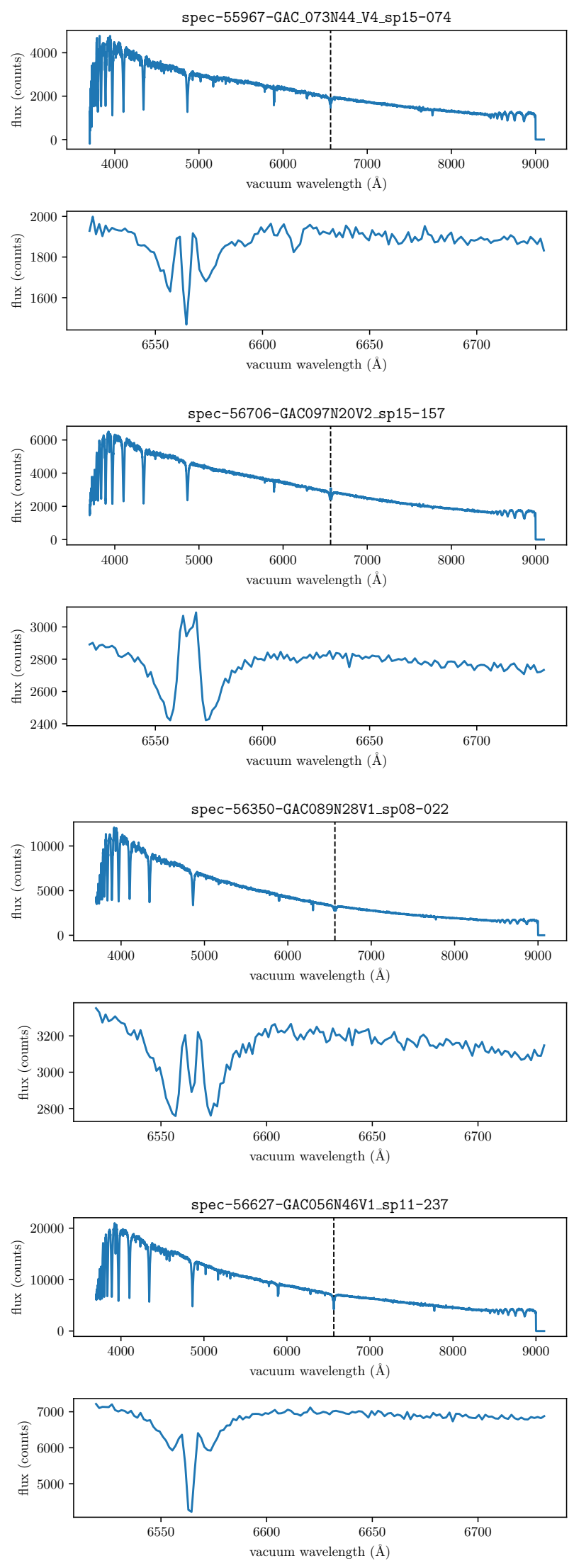

Fig. 7. Examples of spectra classified as double peaks.

absorption with an emission peak in the red wing, and a strong emission combined with absorption at $7600 \AA$ A. A P Cyg profile is visible at $6870 \AA$. 
P. Škoda et al.: Active deep learning in large spectroscopic surveys
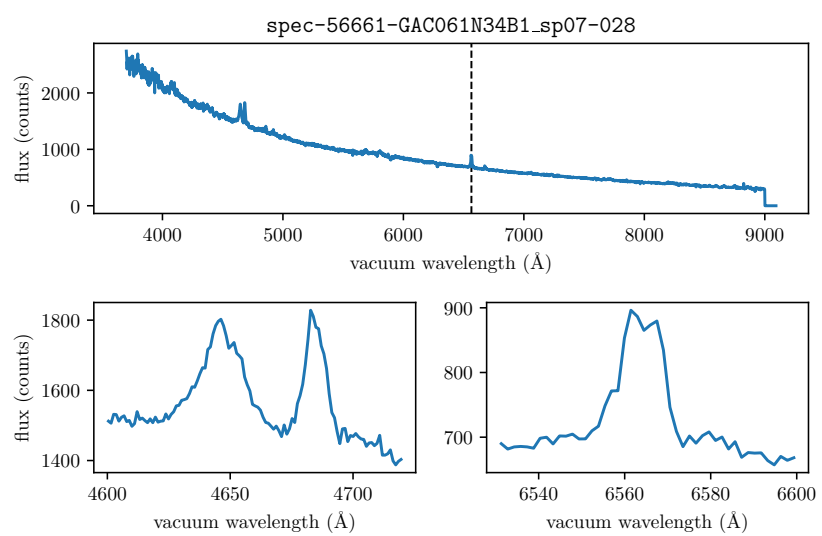

Fig. 8. Candidate Wolf-Rayet WN star LAMOST J040901.83+ 323955.6 .
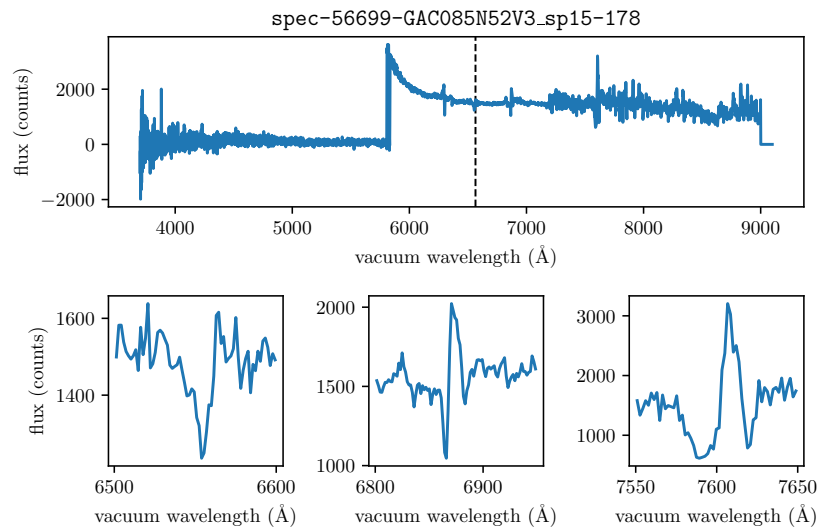

Fig. 9. Object LAMOST J053944.81+531825.7 with a probable gravitation lens.
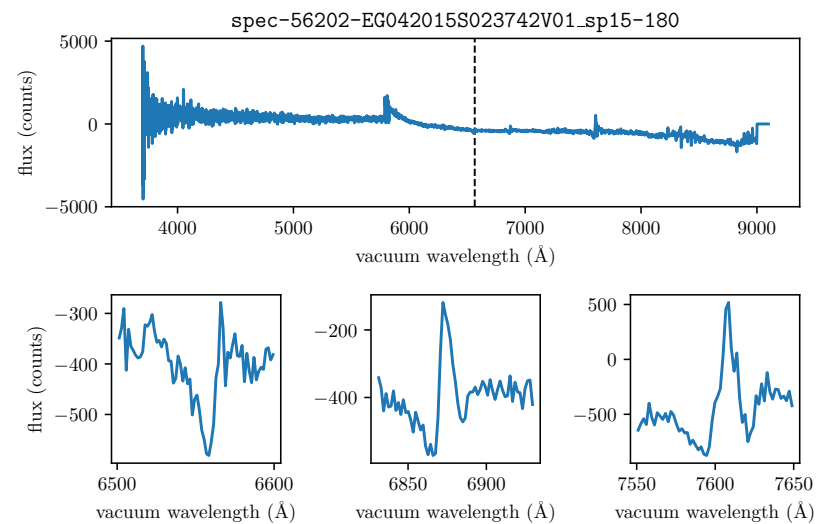

Fig. 10. Object LAMOST J041919.80-020211.6 with complex profiles.

Figure 11 gives examples of other newly discovered objects with complex spectra.

\subsubsection{Supernovae candidates}

Three spectra with a very bright and wide single-peak emission line with an FWHM of about $200 \AA$ are very unusual. H16 identified none of these objects, and they cannot be cross-matched with SIMBAD. We have found all of them in SDSS DR15, but no estimate of the spectral class is given there. They are claimed to be stars, however. Because of their extremely wide red-shifted
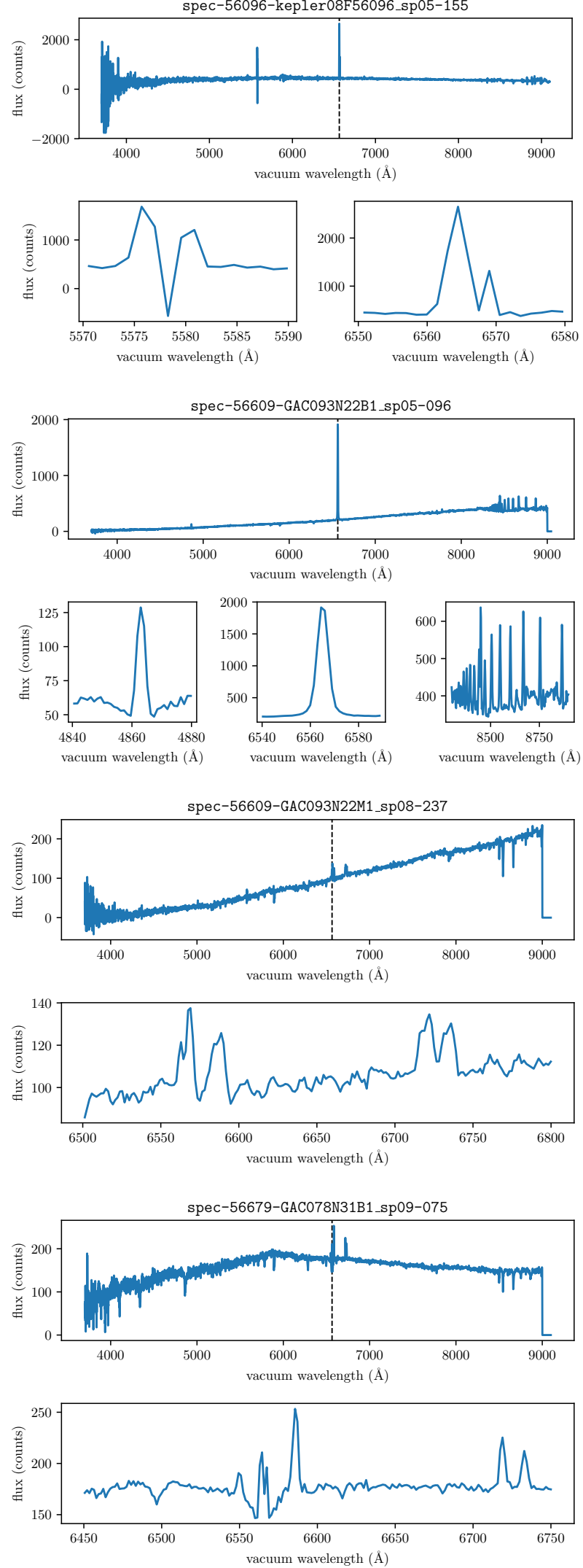

Fig. 11. Random discovered objects with complex profiles.

$\mathrm{H} \alpha$ line (the others are hidden in noise) and because several galaxies are seen around them, we speculate that they may be distant supernovae. However, they might be mere reduction artefacts as well, therefore further investigation is desirable. 


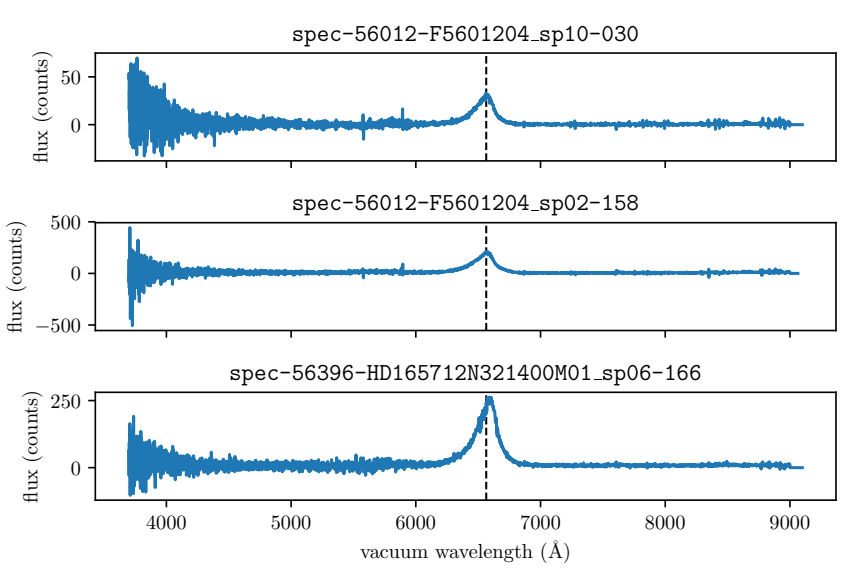

Fig. 12. Supernovae candidates identified in the LAMOST DR2.

Spectrum spec-56012-F5601204_sp10-030 belongs to object LAMOST J114232.73-011535.9. In the SDSS DR15 colour image at this position, a faint white star is visible that may be identified with SDSS J114232.73-011535.9, surrounded by galaxies.

Spectrum spec-56012-F5601204_sp02-158 is the LAMOST J114009.42-012454.3 equivalent to SDSS J114009.42012454.3. A faint orange object is surrounded by a number of galaxies seen within $10^{\prime \prime}$ in the SDSS.

The last spectrum of a similar shape is spec-56396HD165712N321400M01_sp06-166 of object LAMOST J170 $758.50+313441.1$. Its counterpart in the SDSS is seen as a blue circular object, SDSS J170758.50+313441.1, again with the nearby galaxy SDSS J170758.05+313443.6 about 6" apart. The object is also present in the 2MASS and GALEX surveys.

\subsubsection{Detection of extragalactic objects}

Visual inspection of suggested candidates also resulted in the identification of several extragalactic objects, both Seyfert and normal galaxies (confirmed by SIMBAD).

Spectrum spec-56716-HD114322N280318M_sp08-042 belongs to object LAMOST J114631.67+274624.1, which is the Seyfert galaxy 87GB $114356.6+280254$ with redshift 0.3187 .

Spectrum spec-56657-M31020N36M1_sp08-216 belongs to object LAMOST J012555.94+351036.9, which is the Seyfert galaxy 2MASS J01255593+3510368, for which no SDSS spectrum is available.

Spectrum spec-56798-HD141746N331518M01_sp16-150 belongs to object LAMOST J141403.15+352311.3 which is the Seyfert galaxy 2MASX J14140315+3523107. The SDSS spectrum is also available.

Spectrum spec-56752-HD150254N020528B01_sp08-150 belongs to object LAMOST J150711.68+013202.4, which is a star in the outer part of the LINER-type galaxy NGC5850.

Spectrum spec-56304-HD083110N401329F01_sp09-064 belongs to object LAMOST J083426.80+411414.8, which is not in SIMBAD, but it is cross-matched with SDSS DR15 as galaxy SDSS J083426.80+411414.8. An SDSS spectrum is also available.

Spectrum spec-56633-HD095000N333605M01_sp10-109 of object LAMOST J093915.39+331634.2 belongs to galaxy LEDA 2028208 with an available SDSS spectrum.

Spectrum spec-56739-HD114047N212109B sp10-131 of object LAMOST J113114.00+211841.3 belongs to the galaxy LEDA 1647402 with an available SDSS spectrum.

\subsubsection{High-velocity stars}

Identified as target class objects by our CNN (despite the relatively minor deformation of the absorption line profile, which is comparable with a noise level), a group of objects has a considerable red- or blue-shifted $\mathrm{H} \alpha$ line. It appears that most of them are stars labelled in the LAMOST observing program as M31 targets coming from the observing plan that covered the central regions of the M 31 and M 33 galaxies (Luo et al. 2015). The spectra clearly show that the centre of the $\mathrm{H} \alpha$ emission line is heavily blue-shifted, which corresponds to line-of-sight velocities of $-301 \mathrm{~km} \mathrm{~s}^{-1}$ and $-180 \mathrm{~km} \mathrm{~s}^{-1}$ of M 31 and M 33, respectively (van der Marel \& Guhathakurta 2008).

However, other objects are not associated with galaxies by the LAMOST observing plan. The most red-shifted is the spectrum (available in our previews) spec-56357-HD090901 N073047B01_sp12-133 of object LAMOST J091206.52+ 091621.8, which according to the SIMBAD position belongs to the galaxy run-away hypervelocity star LAMOST HVS1 with a stated radial velocity of $611.65 \mathrm{~km} \mathrm{~s}^{-1}$ (Zheng et al. 2014). This spectrum is classified in the LAMOST DR2 archive as a galaxy, while in its FITS header, where it comes from our preview above, as an A0III star. Another spectrum (spec-56285HD090744N104005B03_sp07-117) of the same object, classified in the DR2 archive as an A0III star, is available.

During the visual preview, we found another two objects with high red-shifted radial velocity. Spectrum spec-55997B5599703_sp14-056 of object LAMOST J105350.26+271 352.2, which cannot be identified with SIMBAD, shows RV $331 \mathrm{~km} \mathrm{~s}^{-1}$ in $\mathrm{H} \alpha$ and $293 \mathrm{~km} \mathrm{~s}^{-1}$ in $\mathrm{H} \beta$. Four other spectra of the same star are listed in DR2, namely spec-55910B91005_sp14-056, spec-55998-B5599803_sp14-056, spec56638-HD104953N275826B01_sp08-015, and spec-56685HD104953N275826M01_sp08-015.

Spectrum spec-56617-VB081S05V1_sp02-071 of object LAMOST J052354.52-070508.3 gives radial velocities $358 \mathrm{~km} \mathrm{~s}^{-1}$ in $\mathrm{H} \alpha$ and $351 \mathrm{~km} \mathrm{~s}^{-1}$ in $\mathrm{H} \beta$. It is the same in another spectrum, spec-56617-VB081S05V2_sp02-071.

Spectrum spec-56393-HD172143N395828M01_sp16-162 of object LAMOST J171623.21+412303.1 is quite surprising. The $\mathrm{H} \alpha$ seems to be blue-shifted by about $710 \mathrm{~km} \mathrm{~s}^{-1}$ and the Paschen P13 and P15 lines by $660 \mathrm{~km} \mathrm{~s}^{-1}$ and $630 \mathrm{~km} \mathrm{~s}^{-1}$, respectively. Because of the high noise and apparent asymmetry of the lines, it is impossible to obtain a precision better than about $10 \mathrm{~km} \mathrm{~s}^{-1}$, but it is evident that this star is one of the fastest stars in our galaxy and approaches us, unlike HVS1. However, it may be of extragalactic origin as well, as the image of the object in SDSS DR15 is partly saturated and lies less than 5" away from the large elliptical galaxy. It is known in the GALEX survey as GALEXASC J171623.29+412304.2, and in 2MASS as 2MASS $\mathrm{J} 17162320+4123031$.

\section{Conclusions}

We have introduced a promising method for the discovery of objects of interest in large archives based on active deep learning. This technique, supported by interactive visual classification of a small sample of suggested target classes, is very efficient and has led to the discovery of many new unknown emission-line stars.

To the best of our knowledge, this is the first application of active deep learning techniques in astronomy: used for spectral classification. Many details still need to be elaborated, and more experiments must be run on different samples of various types of spectra. The main advantage of the method is that target 
classes with characteristic spectral features can be identified in cases where the classical deep learning fails because not enough labelled examples are available.

Our experiments identified many emission-line candidates that deserve more detailed examination because they may hide rare astronomical objects with interesting physical properties. All results are publicly available at Zenodo and will also be uploaded in the main astronomical catalogue repository, the CDS Vizier.

Acknowledgements. The early stages of this research were supported by the grant LD-15113 of Ministry of Education, Youth, and Sports of the Czech Republic and the COST Action TD1403 Big Sky Earth. The final parts of the research and all the experiments described here were supported by the same Ministry in project Research Center for Informatics, CZ.02.1.01/0.0/0.0/16_019/0000765 and by the Grant Agency of the Czech Technical University in Prague, grant No. SGS20/212/OHK3/3T/18. Furthermore, this work is based on spectra from the Ondřejov $2 \mathrm{~m}$ Perek telescope and the public LAMOST DR2 survey. Therefore, we would like to thank the Guoshoujing Telescope. Guoshoujing Telescope (the Large Sky Area MultiObject Fiber Spectroscopic Telescope LAMOST) is a National Major Scientific Project built by the Chinese Academy of Sciences. Funding for the project has been provided by the National Development and Reform Commission. LAMOST is operated and managed by the National Astronomical Observatories, Chinese Academy of Sciences. We are namely indebted to Dr. Chenzhou Cui for long-term support of our activities in the framework of collaboration with Chinese VO. Finally, we also express our thanks to Miroslav Šlechta for reduction of all CCD700 spectra used in our research.

\section{References}

Abadi, M., Agarwal, A., Barham, P., et al. 2015, TensorFlow: Large-Scale Machine Learning on Heterogeneous Systems, Software available from tensorflow.org

Alger, M. J., Banfield, J. K., Ong, C. S., et al. 2018, MNRAS, 478, 5547

Alhassan, W., Taylor, A. R., \& Vaccari, M. 2018, MNRAS, 480, 2085

Aniyan, A. K., \& Thorat, K. 2017, ApJS, 230, 20

Bonnarel, F., Fernique, P., Bienaymé, O., et al. 2000, A\&AS, 143, 33

Bromová, P., Škoda, P., \& Vážný, J. 2014, Int. J. Autom. Comput., 11, 265

Chawla, N. V., Bowyer, K. W., Hall, L. O., \& Kegelmeyer, W. P. 2002, J. Artif. Intell. Res., 16, 321

Chollet, F. 2015, Keras, https: //keras. io Accessed: 2 July 2020

de la Calleja, J., Benitez, A., Medina, M. A., \& Fuentes, O. 2011, 2011 International Conference of Soft Computing and Pattern Recognition, 435

Demleitner, M., Neves, M. C., Rothmaier, F., \& Wambsganss, J. 2014, Astron. Comput., 7-8, 27

Domínguez Sánchez, H., Huertas-Company, M., Bernardi, M., Tuccillo, D., \& Fischer, J. L. 2018, MNRAS, 476, 3661

Fernique, P., Allen, M. G., Boch, T., et al. 2015, A\&A, 578, A114

George, D., \& Huerta, E. A. 2018, Phys. Rev. D, 97, 044039

Glorot, X., Bordes, A., \& Bengio, Y. 2011, in 28th International Conference on Machine Learning, eds. L. Getoor, \& T. Scheffer (New York, USA: ACM), 513

Goodfellow, I., Bengio, Y., \& Courville, A. 2016, Deep Learning (MIT Press), http://www . deeplearningbook . org, Accessed: 2 July 2020

Gray, R. O., \& Corbally, C. J. 2009, Stellar Spectral Classification (Princeton University Press)

Gupta, K. D., Pampana, R., Vilalta, R., Ishida, E. E. O., \& de Souza, R. S. 2016, in 2016 IEEE Symposium Series on Computational Intelligence (SSCI), Athens, 1

Hanuschik, R. W., Kozok, J. R., \& Kaiser, D. 1988, A\&A, 189, 147

Heiter, U. 2014, Air-to-vacuum conversion, http://www.astro.uu.se/ valdwiki/Air-to-vacuum20conversion, Accessed: 2 July 2020

Hou, W., Luo, A.-L., Hu, J.-Y., et al. 2016, Res. Astron. Astrophys., 16, 138

Ishida, E. E. O., Beck, R., González-Gaitán, S., et al. 2019a, MNRAS, 483, 2

Ishida, E. E. O., Kornilov, M. V., Malanchev, K. L., et al. 2019b, Active Anomaly Detection for Time-domain Discoveries

Kang, W., \& Lee, S.-G. 2012, MNRAS, 425, 3162

Kingma, D. P., \& Ba, J. 2015, in 3rd International Conference on Learning Representations, eds. Y. Bengio, \& Y. LeCun
Kogure, T., \& Leung, K. C. 2007, in The Astrophysics of Emission-Line Stars (Springer), Astrophys. Space Sci. Libr., 342

Krizhevsky, A., Sutskever, I., \& Hinton, G. E. 2012, in Advances in Neural Information Processing Systems, eds. F. Pereira, C. J. C. Burges, L. Bottou, \& K. Q. Weinberger (Curran Associates, Inc.), 25, 1097

Kurosawa, R., Harries, T. J., \& Symington, N. H. 2006, MNRAS, 370, 580

LeCun, Y., Boser, B., Denker, J. S., et al. 1989, Neural Comput., 1, 541

LeCun, Y., Bengio, Y., \& Hinton, G. 2015, Nature, 521, 436

Lee, Y. S., Beers, T. C., Sivarani, T., et al. 2008, AJ, 136, 2022

Lee, Y. S., Beers, T. C., Carlin, J. L., et al. 2015, AJ, 150, 187

Lin, C.-C., Hou, J.-L., Chen, L., et al. 2015, Res. Astron. Astrophys., 15, 1325

Liu, P., Zhang, H., \& Eom, K. B. 2017, IEEE J. Sel. Top. Appl. Earth Obs. Remote Sens., 10, 712

Luo, A.-L., Zhao, Y.-H., Zhao, G., et al. 2015, Res. Astron. Astrophys., 15, 1095

Luo, A. L., Zhao, Y. H., Zhao, G., et al. 2016, VizieR Online Data Catalog: V/149

Lyon, R. J., Stappers, B. W., Cooper, S., Brooke, J. M., \& Knowles, J. D. 2016, MNRAS, 459, 1104

Nandrekar-Heinis, D., Michel, L., Louys, M., \& Bonnarel, F. 2014, Astron. Comput., 7-8, 37

Pan, S. J., \& Yang, Q. 2010, IEEE Trans. Knowl. Data Eng., 22, 1345

Parimucha, V., \& Škoda, P. 2006, in Binary Stars as Critical Tools \& Tests in Contemporary Astrophysics, eds. W. I. Hartkopf, E. F. Guinan, \& P. Harmanec (Cambridge University Press), Proc. IAU Symp., 240, 486

Podsztavek, O. 2017, Bachelors Thesis, Czech Technical University in Prague, Faculty of Information Technology, https://dspace.cvut.cz/handle/ 10467/69666?locale-attribute=en, Accessed: 2 July 2020

Porter, J. M., \& Rivinius, T. 2003, PASP, 115, 1153

Prati, R. C., Batista, G. E., \& Monard, M. C. 2009, 4th Indian International Conference on Artificial Intelligence, 359

Rastgoo, M., Lemaitre, G., Massich, J., et al. 2016, 3rd International Conference on Bioimaging

Reipurth, B., Pedrosa, A., \& Lago, M. T. V. T. 1996, A\&A, 120, 229

Richards, J. W., Starr, D. L., Brink, H., et al. 2012, ApJ, 744, 192

Russakovsky, O., Deng, J., Su, H., et al. 2015, Int. J. Comput. Vision, 115, 211

Settles, B. 2009, Active Learning Literature Survet, Computer Sciences Technical Report 1648 (Madison: University of Wisconsin)

Silaj, J., Jones, C. E., Tycner, C., Sigut, T. A. A., \& Smith, A. D. 2010, ApJS, 187,228

Simonyan, K., \& Zisserman, A. 2015, in 3rd International Conference on Learning Representations, eds. Y. Bengio, \& Y. LeCun

Škoda, P. 2019, https: //doi .org/10.5281/zenodo. 3242658

Škoda, P., \& Šlechta, M. 2002, Publ. Astron. Inst. Czech. Acad. Sci., 90, 22

Škoda, P., \& Vážný, J. 2012, ASP Conf. Ser., 461, 573

Škoda, P., Draper, P. W., Neves, M. C., Andrešič, D., \& Jenness, T. 2014, Astron. Comput., 7-8, 108

Škoda, P., Bromová, P., Lopatovský, L., Palička, A., \& Vážný, J. 2015, ASP Conf. Ser., 495, 87

Škoda, P., Palička, A., Koza, J., \& Shakurova, K. 2016, Proc. IAU Symp., 325, 180

Škoda, P., Podsztavek, O., \& Tvrdík, P. 2019, https://doi.org/10.5281/ zenodo. 3241520

Solorio, T., Fuentes, O., Terlevich, R., \& Terlevich, E. 2005, MNRAS, 363, 543

Srivastava, N., Hinton, G., Krizhevsky, A., Sutskever, I., \& Salakhutdinov, R. 2014, J. Mach. Learn. Res., 15, 1929

Taigman, Y., Polyak, A., \& Wolf, L. 2017, 5th International Conference on Learning Representations OpenReview. net

Taylor, M. B. 2005, ASP Conf. Ser., 347, 29

Taylor, M. B., Boch, T., \& Taylor, J. 2015, Astron. Comput., 11, 81

Tody, D., Dolensky, M., McDowell, J., et al. 2012, Simple Spectral Access Protocol Version 1.1, IVOA Recommendation 10 February 2012

Traven, G., Zwitter, T., Van Eck, S., et al. 2015, A\&A, 581, A52

van der Marel, R. P., \& Guhathakurta, P. 2008, ApJ, 678, 187

Vilalta, R., Gupta, K. D., Boumber, D., \& Meskhi, M. M. 2019, PASP, 131

Walborn, N. R., \& Fitzpatrick, E. L. 2000, PASP, 112, 50

Waters, C. Z., \& Hollek, J. K. 2013, PASP, 125, 1164

Wu, Y., Luo, A.-L., Li, H.-N., et al. 2011, Res. Astron. Astrophys., 11, 924

Yang, L., MacEachren, A., Mitra, P., \& Onorati, T. 2018, ISPRS Int. J. Geo-Inf., 7

Zeiler, M. D., \& Fergus, R. 2014, in Computer Vision - ECCV 2014, eds. D. Fleet, T. Pajdla, B. Schiele, \& T. Tuytelaars (Cham: Springer International Publishing), 818

Zheng, Z., Carlin, J. L., Beers, T. C., et al. 2014, ApJ, 785, L23

Zickgraf, F.-J. 2003, A\&A, 408, 257 


\section{Appendix A: Comparison with non-active learning}

Table A.1. Results of three runs of non-active learning.

\begin{tabular}{lcc}
\hline \hline \multirow{2}{*}{ Run } & \multicolumn{2}{c}{ Estimated precision (predicted spectrum count) } \\
\cline { 2 - 3 } & Single peak & Double peak \\
\hline No. 1 & $4.1 \%(343988)$ & $2.0 \%(248336)$ \\
No. 2 & $3.0 \%(301396)$ & $2.0 \%(409908)$ \\
No. 3 & $4.0 \%(167545)$ & $0.0 \%(342230)$ \\
\hline
\end{tabular}

Notes. The table shows the precision estimated from a random sample of 100 spectra from each target class, and the numbers in brackets are counts of spectra classified into each target class.

To clarify the real gain of active learning, we compare our active deep learning method to a non-active learning dual scenario. The non-active learning can be considered the zeroth iteration of the application of our active deep learning in Sect. 3. However, the zeroth iteration in our application is carried out in the speededup regime (see the last paragraph of Sect. 3.4).

We carried out an independent experiment to prove the benefits of active learning. We trained our CNN using the setting of the long training with the initial training set of our active deep learning method (the preprocessed Ondřejov dataset), and we used the trained CNN to classify all the spectra in the LAMOST pool. Then, we estimated precision of the $\mathrm{CNN}$ from random samples of 100 spectra from target classes. In order to make a more reliable conclusion, we ran the experiment three times. The results are shown in Table A.1.

The comparison of Tables A.1 and 1 shows that the three CNNs were unable to learn without the support of spectra from LAMOST added to the training set by active learning. Therefore we conclude that the gain of our active deep learning method is significant.

\section{Appendix B: Detailed analysis of emission-line candidates}

All spectra in the candidate list predicted by our $\mathrm{CNN}$ were visually inspected to confirm the correct prediction of their classes. We reviewed not only the limited spectral range around the $\mathrm{H} \alpha$ line given to the CNN (which was only available in the Ondřejov dataset), but also the whole LAMOST spectrum displayed in linear wavelengths (instead of the original logarithmic scale of the input FITS files).

We removed from the candidate spectra those that were clearly bad (reduction artefacts, missing data in the studied spectral range) or were so noisy that the line profile drawn as a continuous line was broken in a sawtooth-like manner. Other bad spectra belonged to cold stars, where the molecular bands, smeared because of the low resolution of LAMOST, mimicked the searched emission profile in a small zoomed range, but the rest of the continua had a similar variability amplitude. The rest were spectra in the non-target class showing only absorption lines without signatures of emission. We removed 58 spectra in total. The list is available in the on-line tables published on Zenodo and CDS. Despite being dropped in the uninteresting (nontarget) class, the visual inspection of apparently bad spectra also yielded several interesting objects, such as high-velocity stars or objects with complicated physics as described in Sects. 4.2.2 and 4.2.4.

In general, it was challenging to decide about the quality of the data and the correct classification in these boundary cases.
We took the size of the line profile disturbance, the fuzziness of spectra (and namely its continua), and other metadata (obtained from the Virtual Observatory as explained below) into account to classify an object as the one with expected emission (e.g. a Be star). Our overall experience with this very subjective classification in boundary cases was, however, very surprising. The deep $\mathrm{CNN}$ was able to see even very tiny signatures of expected shape structures that the human could barely see.

\section{B.1. Technology of the Virtual Observatory}

The verification of the performance of our active deep learning method required many visualisations of spectra with the possibility of previewing entire spectra as well as their zoomed parts. In many cases, we used the positional cross-matching followed by visual inspection of the appearance of a candidate object in common all-sky imaging and photometric surveys. This task would be extremely tedious without the usage of the Virtual Observatory technology based on the IVOA standards, namely the combination of the Table Access (Nandrekar-Heinis et al. 2014) and Simple Spectra Access (Tody et al. 2012) protocols and VO client applications such as TOPCAT (Taylor 2005), Aladin (Bonnarel et al. 2000), or SPLAT-VO (Škoda et al. 2014). All LAMOST DR2 FITS files, converted into the linear wavelength in Angströms, were ingested into a VO server based on the DACHS system (Demleitner et al. 2014) that runs locally in Ondřejov, and the links (called accref or access_url) to individual spectra on that server were joined with spectrum names in the candidate table. This allowed an immediate visualisation and interactive zooming of every spectrum in SPLAT-VO. We were unable to use the original China-VO services because the DR2 is not available via the SSAP protocol (only DR1 is available).

The combination of the TOPCAT, Aladin, and SPLAT-VO tools interlinked using the SAMP protocol (Taylor et al. 2015) allowed us to set up a powerful workflow. In addition to basic operations on tables (e.g. sorting, counting, ordering, and searching in rows) and cross-matching using internal TOPCAT capabilities, we extensively used the CDS cross-match service with SIMBAD running on CDS computers.

The resulting tables were then sent to Aladin and SPLATVO, and the TOPCAT activation actions were set on them, so that the selection of every row in the TOPCAT table triggered a sending operation of the object coordinates to Aladin. Here the detailed image of a star or galaxy from the all-sky surveys DSS2, 2MASS, SDSS, and GALEX quickly appeared thanks to the hierarchical progressive survey (HiPS) technology (Fernique et al. 2015). In addition, the TOPCAT activation action also triggered the sending of accref content to the SPLAT-VO, where the corresponding spectrum was shown. We verified correct cross-matching with SIMBAD by over-plotting all SIMBAD objects that are visible in the field in Aladin (also based on the HIPS catalogues), and placing the pointer at a particular target resulted in showing the SIMBAD web page about the object in a browser. More details are shown in our presentation from the Paris IVOA interoperability meeting (Škoda 2019), which is available on Zenodo ${ }^{7}$.

The productivity of candidate verification was enormously increased by the VO technology in comparison to a manual search for information in multiple sources. It also allowed us to discover the effects described below (e.g. misplacement of an optical fibre). In addition to the VO exploitation, SPLAT-VO was also used for direct measurement of radial velocities through its

\footnotetext{
https://doi.org/10.5281/zenodo. 3242658
} 
P. Škoda et al.: Active deep learning in large spectroscopic surveys

built-in method of line profile mirroring (Parimucha \& Škoda 2006). The vacuum wavelengths of the measured lines were used as reference.

\section{B.2. Multiplicity of exposures}

Some objects in the LAMOST DR2 were observed several times, usually twice in different epochs, but a few spectra in our candidate list were also exposed five times. This fact may be used for analysing the evolution of the line profiles, which is typical for some Be stars. For example, the comparison of spectrum spec-56625-GAC113N37V1_sp07-098 of object LAMOST J074244.51+353401.3 exposed 281 days after the spec-56344-GAC113N37V1_sp07-098 shows a doublepeak emission that diminishes in a deep absorption line, while the spectrum spec-56350-GAC089N28V1_sp04-121 of object LAMOST J055821.00+284549.6 exposed 474 days after the spectrum spec-55876-GAC_089N28_B2_sp04-121 shows the blue-shifted peak of a double-peak profile that decreases, while the red-shifted peak is stable and the absorption in the line core is much deeper.

We grouped 855 of 4379 candidate spectra into 398 groups with the same designation as stated in the LAMOST header. The remaining 3524 objects seemed to have only one exposure. However, the internal cross-matching using the coordinates in the radius of $5^{\prime \prime}$ and visual verification of spectra shape identified several objects with a different designation that lie close together. We were able to identify 436 groups of multiple exposures within a radius of $5^{\prime \prime}$. However, this complicates the cross-matching because we cannot consider the object designation as a unique identifier. For example, objects LAMOST J053611.80+273436.0 and LAMOST J053611.79+273435.9 are the same, as are LAMOST J041417.60+280609.6, LAMOST J041417.61+280609.5, and LAMOST J041417.62+280609.4.

We also found a case of two different stars with almost identical spectra: spectra spec-56204-GAC080N33B101_sp08234 of star LAMOST J052402.81+334101.7 and spec-563 06-GAC080N33B2_sp08-234 of star LAMOST J052401.53+ 334120.9 exhibit almost identical $\mathrm{H} \alpha$ emission, although the stars are 24" apart and have different SIMBAD names, 2MASS J05240280+3341017 and 2MASS J05240153+3341210. We suppose that the two spectra are contaminated by the emission from the gaseous nebula around them, or (see similar cases below) the spectrum of one star might be mixed with emission light of the second star before entering the fibre.

We still preserved the list of misclassified spectra (58 in total, visually confirmed to be bad spectra or with an incorrect class, namely absorption) because they proved to be useful for the discovery of some peculiar objects.

\section{B.3. LAMOST classification pipeline flaws}

The LAMOST FITS headers contain the estimate of the spectral type of almost all stars as well as labels produced by the automatic pipeline (Wu et al. 2011) that mark the objects as nonstellar (galaxy, quasar, or unknown). However, we noted various inconsistencies in these labels. For example, object LAMOST J053040.90+260534.6 is classified in spectrum spec-56218GAC083N27B2 sp02-234 as star B6, while in spec-56271GAC084N26B1_sp10-057 it is A2V, although the visually examined line profiles look very similar. Although some stars are typical Be stars (identified by $\mathrm{H} 16$ as classical Be stars), the LAMOST classification mostly assigns class A, but also class F or G. Objects marked as "Non" are often just bad data with reduction artefacts, but there are also some very interesting cases, as was shown in previous sections.

\section{B.4. Comparison with $\mathrm{H} 16$}

The main reason why we have used the LAMOST DR2 survey for our analysis instead of publicly available later versions (e.g. DR4 with 7.6 million spectra is available since July 2018) is not the lower data volume (which facilitates the whole analysis), but the excellent opportunity of comparing our active deep learning methods with the more straightforward pattern-recognition algorithm of $\mathrm{H} 16$ on the same data set. Their catalogue gives a more detailed line profile classification into six classes (obtained by cross-correlation with 27 templates) and attempts to cross-match the objects with other known emission-star catalogues, namely, with SIMBAD.

The detailed analysis of the cross-matching with our candidate list has, however, identified a number of problems that prevented us from using their catalogue for a direct comparison of the performance of our method with theirs, despite the same data set and the same target class (emission-line objects).

The first discrepancy is the non-unique identification of objects. As they do not give spectrum identifications but only an object designation, we joined our candidate list with their catalogue using the LAMOST designation (which is in fact an encoding of coordinates in J2000). However, the coordinate cross-matching using circles with a radius of $3^{\prime \prime}$ identified objects with coordinates stated by H16 that were different from those in the LAMOST header. For example, for object LAMOST J015611.38+580928.6, H16 gives a position that is $2.4^{\prime \prime}$ offset from those in the FITS header. As the object $\mathrm{J} 034031.33+504451.4$ gives the largest coordinate discrepancy of $4.96^{\prime \prime}$ between coordinates in the H16 catalogue and the LAMOST header, we cross-matched our candidates with H16 with a radius of $5^{\prime \prime}$, instead of using just the verbatim match of designations. We assume that the differences in coordinates stated in H16 and those we obtained from the FITS header may be caused by some post-processing of the LAMOST DR2 archive before it was publicly released (H16 probably used the DR2 before its public release).

We also visually verified randomly selected spectra of objects from their catalogue (using the multiple SSA query in TOPCAT at the given coordinates) and realised that many objects in their catalogue were not justified to have the emission signatures in $\mathrm{H} \alpha$ line, as the apparent emission bumps on line profiles were just a coincidence of a noise fluctuations, similar to what we discuss at the beginning of Appendix B. This concerns most of the objects that are classified by them as unknown. For example, the spectrum of LAMOST J010450.45+423607.2 is completely spoiled by reduction artefacts that introduce a forest of narrow spikes everywhere. Both spectra spec-55976-GAC_084N40_V1_sp07095 and spec-56257-GAC089N38V1_sp14-033 of LAMOST $\mathrm{J} 054458.54+382954.3$ are noisy as well.

Many unknown types are probably not stars at all. For example, object LAMOST J000943.24+495009.2 is, in fact, the galaxy LEDA 2354919. Many objects of 1919 marked by H16 as unknown are therefore probably not well justified as emissionline stars.

The same problem concerns 3597 objects marked as the H II region. For example, all four available spectra spec-566 18-GAC057N34B1_sp06-069, spec-56661-GAC061N34B1_ sp03-203, spec-56680-GAC057N34B2_sp06-069, and spec56685-GAC061N34B2_sp03-203 of object LAMOST J040 
$124.35+343559.0$ lack a signature of nebula emission lines, and the apparent bump in the $\mathrm{H} \alpha$ core is just coincidence of noise (compared with the noise amplitude in the continuum).

The situation is not better for 5580 stars marked by H16 as $\mathrm{CBe}$ type, the classical Be stars. Here, a random visual inspection identified a number of spectra that are too noisy to be able to assess the profile or spectra without any emission signatures. For example, the single-spectrum objects J000414.96+ 463022.6, J000955.98+392419.0, J003506.02+271339.9 and J004201.02+433802.0 that were classified by $\mathrm{H} 16$ as CBe with $\mathrm{H} \alpha$ type II profile or J052630.97+291516.9 with line profile classified as type VI are completely noisy.

For objects with multiple exposures, one spectrum is often extremely noisy (but because of the noise fluctuation, the profile may be judged as having some tiny emission in absorption), while the others show clearly only a pure absorption profile. This seems to be the case of many CBe type II profiles (e.g. spectra spec-55880-B8004_3_sp03-189 and spec-55930-B55 93002_sp03-192 of object J024156.88+535432.5) or CBe type VI (e.g. spectra spec-56350-GAC089N28V2_sp04-081 and spec-56684-GAC089N28V2_sp04-081 of object J055836.34+ 283405.1, spectra spec-55875-B7505_sp14-198 and spec55910-B91003_sp14-198 of object J015840.60+583322.6, or spectra spec-55879-B7905_1_sp01-140 and spec-55910B91003_sp01-140 of object J021505.52+565827.8).

We also found objects where all spectra are just extremely noisy. For example, this is a case of spectra spec-56627HD095359N274143M01_sp06-068 and spec-56687-HD101 242N281431M_sp10-078 of object J100140.14+274030.6.

Taking all the uncertainties described above into consideration, we were unable to estimate the performance of our method by a direct comparison with H16, as many of their objects with reported emission are not well justified. After some experiments, we had 2644 spectra that were identified by our active deep learning procedure that were also justified to be discovered by H16.

\section{B.5. Cross-matching with SIMBAD}

As many objects in our candidate list look interesting because they resemble Be stars, cataclysmic variables, or young stellar objects, it is important to find more information about them. We therefore tried to cross-match them with the SIMBAD database using the CDS cross-match service built-in the TOPCAT. It is based on finding the minimum angular distance between object coordinates and the SIMBAD catalogue in a small circle of a given radius on the sky. As object coordinates, we took the coordinates given in the LAMOST FITS headers, which represent the coordinates on which the LAMOST optical fibre was placed.

The simple idea of cross-matching using tight tolerance (of the order $1-2^{\prime \prime}$ ) appeared to be incorrect when we started to verify the given position in the all-sky surveys DSS2, 2MASS, and SDSS (using the Aladin sky atlas). We noted faint spectra of objects with a prominent emission profile on coordinates, where there was no visible object in the sky surveys, but there were bright stars nearby, for which SIMBAD stated a young star or directly the emission-line object. The spectra in some fibres probably came from the bright object at a distance of even tens of arcseconds from the fibre position. We therefore finally crossmatched our list of candidates with SIMBAD in different circles of sizes 5" up to $300^{\prime \prime}$ and inspected the SIMBAD type of
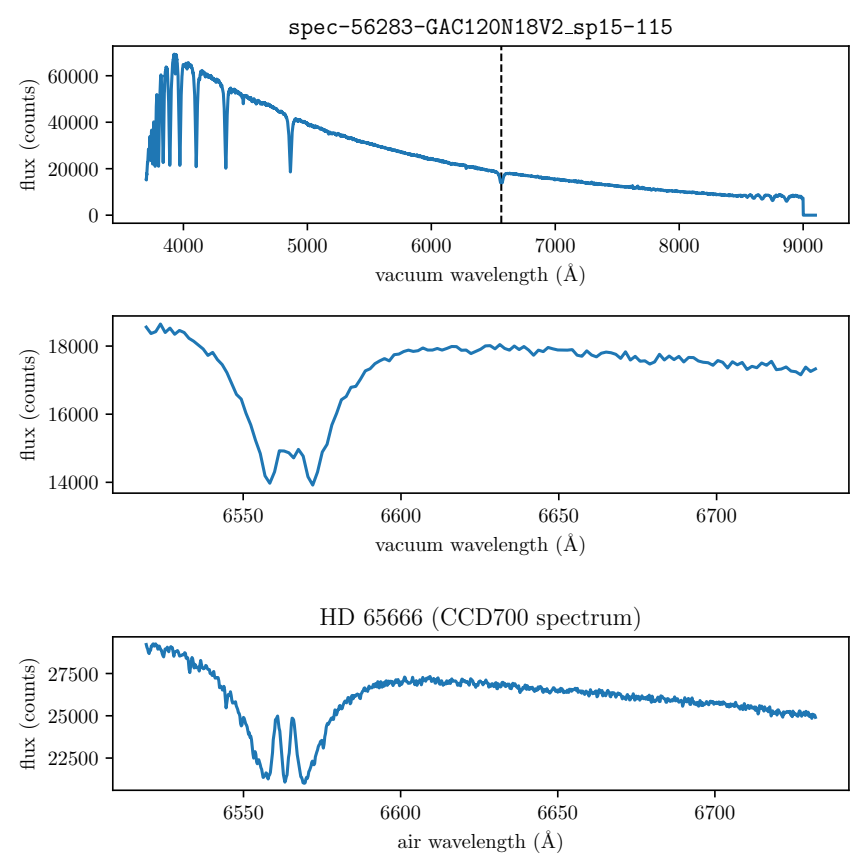

Fig. B.1. Spectrum of the star that is cross-matched as HD 65666 . The upper two panels are from LAMOST, and the lower panel is from the CCD700 archive of the Perek $2 \mathrm{~m}$ telescope. It is a bright star: $V=$ $7.1 \mathrm{mag}$. However, the LAMOST coordinates are offset by $6.5^{\prime \prime}$, so it is difficult to cross-match it with SIMBAD.

objects with larger distances until we confirmed that the match was incorrect (the nearest SIMBAD objects was not of emission nature). The number of cross-matched objects also started to rise steeply after a certain tolerance was met, indicating that the nearest object was not the correct one. After some iterations, we set the acceptable radius for a SIMBAD cross-match to $20^{\prime \prime}$, and we visually inspected that the objects we cross-matched with SIMBAD were reasonably bright targets of emission type. In several cases, we found a galaxy or a nebula (and the corresponding cross-matched object was confirmed by viewing at its spectrum).

Here are few examples of misplaced light entering the fibre: spectrum spec-56618-GAC105N47V2 sp10-151 of object LAMOST J065632.72+461614.9 is the spectrum of star Psi 9 Aur offset by 13", or spec-56295-VB056N24V1_sp08031 of object LAMOST J034912.80+240820.0 belongs to the bright (5 mag) Be star 28 Tau (Pleione), which is located $22^{\prime \prime}$ away from the fibre position.

The spectra with a prominent emission spec-55960GAC_101N09_V1_sp10-152, spec-55960-GAC_101N09 V2_sp10-152 and spec-55968-GAC_101N09_V3_sp10-15 of object LAMOST J063910.49+084435.4 are probably emitted by nebula around the Herbig Ha/Be star R Mon, which lies 26" away.

A very interesting case was also found for spectrum spec-56283-GAC120N18V2_sp15-115 of object LAMOST J080032.50+185028.9, which is marked by the LAMOST pipeline as an A1V star with $r_{\text {mag }}$ of value 11.04 (not identified in H16). It is the bright seventh magnitude star HD 65666 with a fibre offset by $6.5^{\prime \prime}$. Figure B.1 also shows its spectrum obtained by the Ondřejov $2 \mathrm{~m}$ Perek Telescope with a spectral resolution 13000 , which unveils a more complex structure of the line profiles that is typical for Be stars. 